\title{
Treatment of Pathological Fractures of the
} Proximal Femur Due to Advanced Metastasis of Highly Malignant Tumors: A Clinical Controlled Study of Enterostenosis Reconstruction and Conservative Treatment

\author{
Weikun Zheng' ${ }^{1}$ Junfen Tang ${ }^{2}$, Wende Xiao ${ }^{1}$, Weishan Cai ${ }^{1}$ \\ ${ }^{1}$ Department of Orthopedics, Guangzhou First People's Hospital, Guangzhou, China \\ ${ }^{2}$ Department of Neurology, Guangzhou First People's Hospital, Guangzhou, China \\ Email: sysys606@163.com
}

How to cite this paper: Zheng, W.K., Tang, J.F., Xiao, W.D. and Cai, W.S. (2020) Treatment of Pathological Fractures of the Proximal Femur Due to Advanced Metastasis of Highly Malignant Tumors: A Clinical Controlled Study of Enterostenosis Reconstruction and Conservative Treatment. Journal of Biosciences and Medicines, $\mathbf{8}$, 127-137.

https://doi.org/10.4236/jbm.2020.88012

Received: July 17, 2020

Accepted: August 16, 2020

Published: August 19, 2020

Copyright $\odot 2020$ by author(s) and Scientific Research Publishing Inc. This work is licensed under the Creative Commons Attribution International License (CC BY 4.0).

http://creativecommons.org/licenses/by/4.0/ (c) (i) Open Access

\begin{abstract}
Objective: To investigate whether different treatment methods have an impact on the quality of life and life span after fracture of patients with proximal femoral pathological fractures caused by advanced metastasis of highly malignant tumors. Methods: Karnofsky performance status (KPS) and visual analogue score (VAS) were counted at the time of admission and 2 months after the treatments. Survival analysis was implemented to compare the median survival time and 6-month survival rate of the 2 groups. Musculoskeletal score (MSTS) was used to evaluate limb function in the surgical group at 2 months after the treatment. Results: There was no significant difference in KPS score and VAS score between the two groups at the time of admission $(p>0.05)$. At 2 months after treatment, the KPS score of the surgical group was higher than that of the conservative group $(\mathrm{P}<0.05)$, and the VAS score of the survivors of the surgical group was lower than that of the conservative group $(\mathrm{P}<0.05)$. Survival analysis showed that the median survival time and 6-month survival rate after fracture in the surgical group were higher than those in the conservative group $(\mathrm{P}<0.05)$. After 2 months of treatment, the average MTST score of survivors in the surgical group was $20.38 \pm 0.9$ (16 26 points). Conclusion: Surgical intervention can benefit patients with pathological fractures of the proximal femur due to metastasis of highly malignant tumors in terms of quality of life and survival. Local tumor resection and endoparasitic replacement, which can be tolerated by most patients, can effectively reconstruct the limb function of these patients and restore their self-care ability.
\end{abstract}




\section{Keywords}

Malignant Tumors, Proximal Femoral Pathological Fracture, Endoparasitic Proximal Femoral Replacement, Conservative Treatment

\section{Introduction}

Advanced bone metastases from malignant tumors are very common, especially breast cancer, lung cancer, and gastric cancer. The common metastatic sites are the spine and lower extremities. Malignant bone metastases of the lower extremities mostly occur in the hips, especially the proximal femur, which is likely to cause the proximal femur pathological fractures [1], which are important factors for patients with poor prognosis [2]. At present, a large number of scholars [1] [3] [4] believe that as long as the patient's life expectancy is not too short $(>2$ months), surgical measures should be used to improve the quality of life; among them, endoparasitic proximal femoral replacement is an important technique [5] [6]. However, in China, there are still a large number of patients and their families choose conservative treatment after pathological fracture of the proximal femur caused by metastases of tumor, especially the highly malignant tumors such as lung cancer and breast cancer. In addition, most of the domestic studies on patients with this type of fracture are descriptive studies of a single technique (such as a certain internal fixation method), and there is a lack of randomized controlled studies grouped by different treatments. This study conducted a retrospective case-control study on patients with proximal femoral pathological fractures caused by bone metastases from highly malignant tumors in our hospital from 2014 to 2019. The purpose of this retrospective case-control study was to: 1 ) investigate if different treatments have the impact on the quality of life and life span of patients after fracture, and to provide a scientific reference for the selection of clinical treatment and patient guidance; 2) evaluate the feasibility, effectiveness and safety of local tumor resection united endoparasitic proximal femoral replacement in reconstructing the limb function of such patients.

\section{Methods}

\subsection{Clinical Data}

Patients with pathological fractures of the proximal femur caused by bone metastases from malignant tumors treated in our hospital from 2014 to 2019 were observed. Inclusion criteria: 1) A clear history of primary malignant tumor; 2) Fracture, confirmed by auxiliary examination, of the proximal femur cause by slight external force, or no obvious external force is found; 3) CT, MR, bone Scanning clearly showed tumor cell invasion at the fracture site; 4) According to the evaluation of the Dutch model [7], the median survival time is more than 3 months; cardiopulmonary function evaluation, orthopedic evaluation and anesthesia evaluation shows the patient can tolerate the surgery 5) The patient or 
their families sign acceptance in the test consent form and was approved by the hospital ethics committee. Exclusion criteria: 1) The primary tumors are low-grade malignant tumors such as thyroid cancer and prostate cancer, and the expected survival period is too long; 2) The patient accompanying stroke, dementia, mental illness, etc. who can not cooperate with the investigator.

\subsection{Grouping and Intervention}

All the research subjects and their families were informed of the detailed medical information base on the fracture situation and the oncology specialty. We analyzed the pros and cons of the operation for them, emphasizing the feasibility and necessity of the operation, but respecting the patient's final treatment choice. Finally, 31 patients were included in the study and divided into 2 groups according to the final fracture treatment methods: 1) 14 patients in the surgical group were treated with tumor resection united endoparasitic reconstruction of the proximal femur (or combined acetabulum); 2) 17 patients in the conservative group. After the disease notification operation education, the patient's willingness of conservative treatment was respected and conservative treatment was given, and a notice of illness was signed. All patients were simultaneously consulted in the oncology department or transferred to the oncology department opportunely for oncology treatment. According to the guide and the actual situation of the patient, strict and appropriate oncology treatment was performed. Surgical process: 1) local tumor resection. The patient was lying in the lateral position and a contralateral incision of the skin was made. Incision of fascia lata, entering from the gap between the posterior thigh muscle group and the lateral femoral muscle, splitting the gluteus maximus, cutting the external rotation muscle group, and cutting off the switch sac from the contralateral hip joint to expose the femoral head and tumor segment. Remove the femoral head and the tumor segment according to the preoperative, the distal range reaches more than $2 \mathrm{~cm}$ of normal tissue, and intraoperatively send frozen pathology was performed to confirm the complete excision of the tumor segment. Combined with the auxiliary preoperative examination, explore the acetabulum for tumor invasion. If the proximal femur is singly violated, the joint capsule can be retained. If the pelvis is violated at the same time, the joint capsule is removed, the pelvic tumor is scraped, and the bone cement is filled, and total hip replacement would be performed if necessary. If the soft tissue were invaded at the same time, it would be also removed, but try to keep the lateral muscle groups. 2) prosthesis installation. Measure the femur length, femur head size, etc. Depending on the completeness of acetabulum, a tumor-type double-action half-hip or total hip replacement would be adopted. After the trial installation of the prosthesis, the matching degree, the length of the lower extremities, the tension and the range of motion of the hip joint should be checked. The flexion, adduction and internal rotation must be stable. The bone cement is pre-injected into the medullary cavity, and the customized prosthesis is implanted and solidified by keeping the incline $10-15$ forward. 3) Stabilize joints and reconstruct abduction. After the 
prosthesis is cured, if the joint capsule remains, it can be closed and sutured on the neck of the prosthesis to strengthen the stability of the hip joint. Fixing the remaining abductor and mid gluteal muscle stops at the trochanter. The lateral femoral muscle rotates to cover the fixed point, and its front is sutured with the external femoral muscle. If there is no remaining joint capsule, anti-rotation shoes and braces need to be born shortly after operation. Conservative treatment: patients in the conservative group were told to have a rest on the bed and local skin traction according to body weight was performed. We pay great attention to prevent the complications of and bed rest by strengthening precaution such as turning over and patting the back.

\subsection{Evaluation}

All patients were followed up for life. Karnofsky performance status (KPS) and visual analogue score (VAS) were counted at the time of admission and 2 months after the treatments. Survival analysis, focus on death, was implemented to compare the median survival time and 6-month survival rate of the 2 groups. Musculoskeletal score (MSTS) was used to evaluate limb function in the surgical group at 2 months after the treatment.

\subsection{Statistical Processing}

All data were processed by SPSS20.0 statistical software, measurement data comparison was by $t$ test, count data comparison was by $t$ test, survival comparison was by Kaplan-Meier survival analysis, and $\mathrm{P}<0.05$ was considered statistically significant.

\section{Results}

General clinical data and admission evaluation of all patients are shown in Table 1.

\section{Outcomes:}

The 14 patients in the surgical group, 13 patients achieved full load after operation, and the time of starting weight-bearing was 1 week and the time of full load was 2 - 4 weeks; 1 patient was complicated with cardiopulmonary failure after surgery at 36 days after surgery, who died of multiple organ failure and failed to achieve weight bearing. (2) of the 17 patients in the conservative group. 5 patients achieved partial weight-bearing until the end of follow-up. The time for partial weight-bearing was 8 - 9 weeks. 12 patients remained in bed or wheelchair transfer until death. None of the conservative group achieves full weight-bearing. Three patients in the conservative group died within 2 months and did not achieve a 2-month follow-up after fracture. Detail outcome was shown in Table 2.

At 2 months after treatment, the KPS score of the surgical group was higher than that of the conservative group $(61.43 \pm 19.56$ vs. $27.64 \pm 17.15, \mathrm{P}<0.05)$, and the VAS score of the survivors of the surgical group was lower than that of the conservative group $(2.92 \pm 0.64$ vs. $5.79 \pm 0.89, \mathrm{P}<0.05)$. Figure 1 showed 
Table 1. General clinical information and admission evaluation of patients.

\begin{tabular}{|c|c|c|c|c|c|c|c|}
\hline Number & Group & Gender & Age & Fracture site & Primary tumor & $\begin{array}{l}\text { KPS while } \\
\text { admitted }\end{array}$ & $\begin{array}{l}\text { VAS while } \\
\text { admitted }\end{array}$ \\
\hline 1 & 1 & $\mathrm{~F}$ & 58 & $\mathrm{~L}$ & liver & 30 & 7 \\
\hline 2 & 1 & $\mathrm{~F}$ & 64 & $\mathrm{~L}$ & gastric & 40 & 8 \\
\hline 3 & 1 & M & 71 & $\mathrm{R}$ & lung & 30 & 7 \\
\hline 4 & 1 & $\mathrm{~F}$ & 68 & $\mathrm{~L}$ & breast & 30 & 7 \\
\hline 5 & 1 & M & 62 & $\mathrm{~L}$ & colorectal & 40 & 6 \\
\hline 6 & 1 & $\mathrm{M}$ & 76 & $\mathrm{~L}$ & lung & 30 & 7 \\
\hline 7 & 1 & M & 59 & $\mathrm{R}$ & gastric & 30 & 7 \\
\hline 8 & 1 & $\mathrm{~F}$ & 67 & $\mathrm{~L}$ & breast & 30 & 7 \\
\hline 9 & 1 & M & 66 & $\mathrm{R}$ & lung & 30 & 7 \\
\hline 10 & 1 & M & 74 & $\mathrm{~L}$ & gastric & 40 & 6 \\
\hline 11 & 1 & $\mathrm{~F}$ & 65 & $\mathrm{~L}$ & breast & 40 & 7 \\
\hline 12 & 1 & M & 71 & $\mathrm{~L}$ & lung & 30 & 7 \\
\hline 13 & 1 & $\mathrm{~F}$ & 66 & $\mathrm{R}$ & colorectal & 30 & 7 \\
\hline 14 & 1 & M & 76 & $\mathrm{~L}$ & lung & 30 & 8 \\
\hline 15 & 2 & $\mathrm{~F}$ & 77 & $\mathrm{~L}$ & breast & 30 & 7 \\
\hline 16 & 2 & M & 67 & $\mathrm{R}$ & liver & 30 & 6 \\
\hline 17 & 2 & $\mathrm{~F}$ & 73 & $\mathrm{~L}$ & lung & 40 & 7 \\
\hline 18 & 2 & M & 70 & $\mathrm{~L}$ & lung & 30 & 8 \\
\hline 19 & 2 & $\mathrm{~F}$ & 68 & $\mathrm{~L}$ & breast & 30 & 7 \\
\hline 20 & 2 & $\mathrm{~F}$ & 65 & $\mathrm{R}$ & lung & 30 & 6 \\
\hline 21 & 2 & M & 72 & $\mathrm{~L}$ & lung & 40 & 7 \\
\hline 22 & 2 & M & 60 & $\mathrm{~L}$ & gastric & 30 & 7 \\
\hline 23 & 2 & M & 57 & $\mathrm{~L}$ & lung & 30 & 6 \\
\hline 24 & 2 & F & 78 & $\mathrm{R}$ & liver & 30 & 7 \\
\hline 25 & 2 & M & 61 & $\mathrm{~L}$ & gastric & 30 & 7 \\
\hline 26 & 2 & $\mathrm{~F}$ & 72 & $\mathrm{~L}$ & breast & 30 & 8 \\
\hline 27 & 2 & M & 74 & $\mathrm{R}$ & lung & 30 & 6 \\
\hline 28 & 2 & F & 62 & $\mathrm{~L}$ & breast & 30 & 7 \\
\hline 29 & 2 & $\mathrm{~F}$ & 77 & $\mathrm{R}$ & colorectal & 40 & 6 \\
\hline 30 & 2 & M & 61 & $\mathrm{~L}$ & lung & 30 & 7 \\
\hline 31 & 2 & $\mathrm{M}$ & 67 & $\mathrm{~L}$ & liver & 30 & 7 \\
\hline
\end{tabular}

Table 2. Detail outcomes of the two groups.

\begin{tabular}{ccccccc}
\hline Number & Group & KPS at 2M & $\begin{array}{c}\text { VAS at 2 M of } \\
\text { survivor }\end{array}$ & $\begin{array}{c}\text { Survival time after Partial weight } \\
\text { fracture (M) }\end{array}$ & bearing (W) & Full load (W) \\
\hline 1 & 1 & 50 & 3 & 6 & 1 & 3 \\
2 & 1 & 70 & 3 & 18 & 1 & 3 \\
\hline
\end{tabular}




\section{Continued}

\begin{tabular}{|c|c|c|c|c|c|c|}
\hline 3 & 1 & 60 & 3 & 9 & 1 & 3 \\
\hline 4 & 1 & 50 & 4 & 25 & 1 & 3 \\
\hline 5 & 1 & 70 & 3 & 13 & 1 & 2 \\
\hline 6 & 1 & 50 & 3 & 6 & 1 & 4 \\
\hline 7 & 1 & 70 & 2 & 14 & 1 & 3 \\
\hline 8 & 1 & 70 & 3 & $>28$ & 1 & 4 \\
\hline 9 & 1 & 70 & 3 & 7 & 1 & 3 \\
\hline 10 & 1 & 60 & 4 & 17 & 1 & 4 \\
\hline 11 & 1 & 80 & 2 & 31 & 1 & 3 \\
\hline 12 & 1 & 70 & 2 & 6 & 1 & 3 \\
\hline 13 & 1 & 70 & 3 & 17 & 1 & 3 \\
\hline 14 & 1 & 0 & & 2 & & \\
\hline 15 & 2 & 20 & 6 & 4 & & \\
\hline 16 & 2 & 30 & 6 & 3 & & \\
\hline 17 & 2 & 0 & & 2 & & \\
\hline 18 & 2 & 20 & 7 & 3 & & \\
\hline 19 & 2 & 20 & 7 & 9 & 8 & \\
\hline 20 & 2 & 30 & 6 & 3 & & \\
\hline 21 & 2 & 20 & 7 & 5 & & \\
\hline 22 & 2 & 50 & 5 & 10 & 8 & \\
\hline 23 & 2 & 50 & 5 & 6 & & \\
\hline 24 & 2 & 0 & & 2 & & \\
\hline 25 & 2 & 50 & 6 & 16 & 9 & \\
\hline 26 & 2 & 30 & 6 & 8 & 9 & \\
\hline 27 & 2 & 20 & 5 & 4 & & \\
\hline 28 & 2 & 50 & 4 & 13 & 8 & \\
\hline 29 & 2 & 0 & & 1 & & \\
\hline 30 & 2 & 30 & 5 & 4 & & \\
\hline 31 & 2 & 30 & 6 & 3 & & \\
\hline
\end{tabular}

that the median survival time and 6-month survival rate after fracture in the surgical group were higher than those in the conservative group (13M vs. $4 \mathrm{M}$, $85.7 \%$ vs. $35.3 \%, \mathrm{P}<0.05)$. Comparison and analysis of data between and within the two groups were shown in Table 3.

In the surgical group, 8 patients underwent femoral tumor segment resection united prosthesis replacement, 6 patients underwent femoral tumor segment and pelvic local lesion resection united prosthesis replacement and bone cement local filling, 13 patients survived after 2 months of the fracture. The MSTS score was $16-26$, with an average score of $20.38 \pm 0.93$. One patient had poor gait and was corrected through rehabilitation exercise and brace wear. No infection, loose prosthesis, dislocation, fractures around the prosthesis was found. 


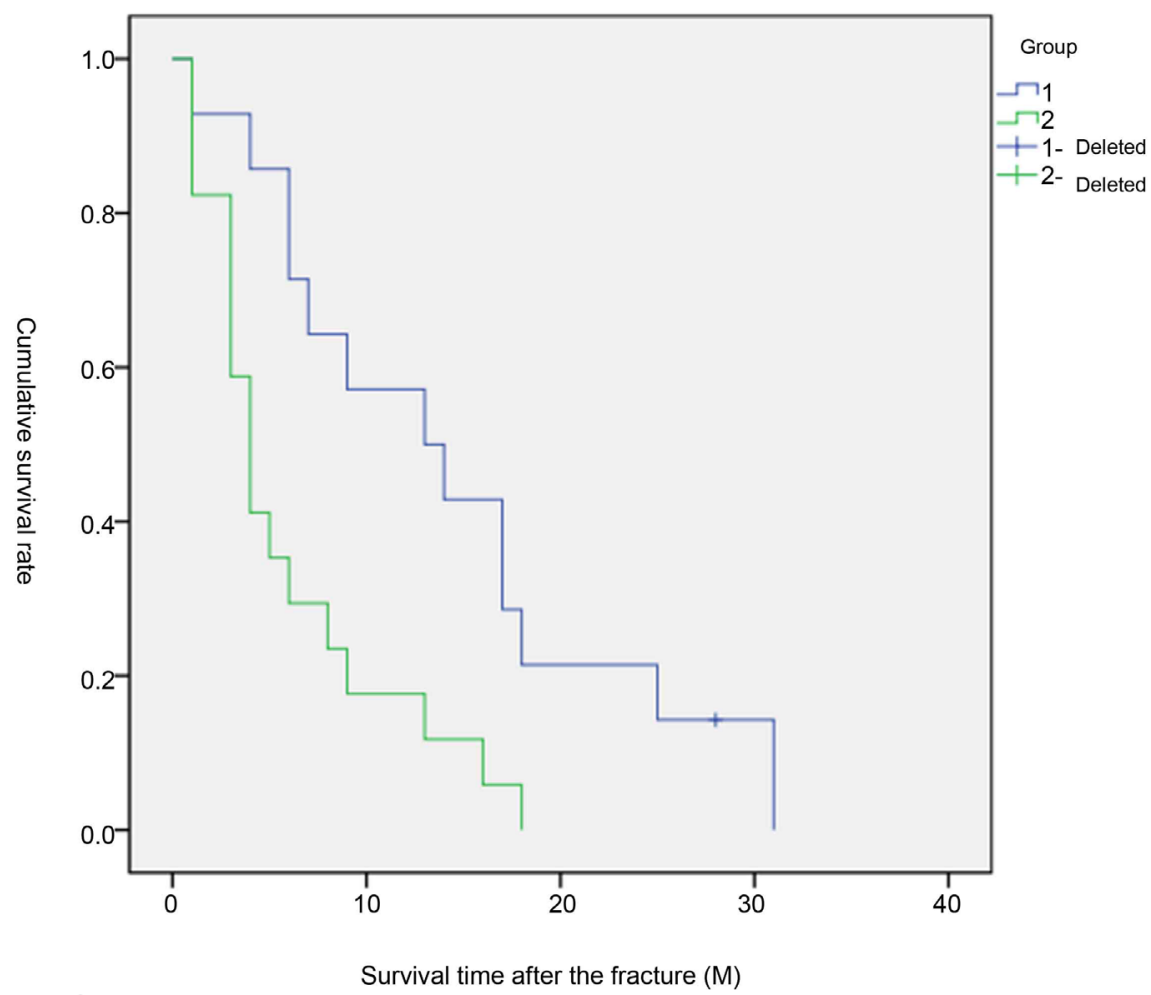

Figure 1. Survival analysis after fracture of the 2 groups, 1: surgical group, 2: conservative group.

Table 3. Comparison and analysis of data between and within two groups.

\begin{tabular}{cccccccccc}
\hline \multirow{2}{*}{ Group } & \multicolumn{3}{c}{ KPS } & \multicolumn{3}{c}{ VAS } & \multicolumn{2}{c}{ Survival rate at } \\
\cline { 2 - 9 } & White admitted & at $2 \mathrm{M}$ & t value & P value & White admitted & at $2 \mathrm{M}$ & t value & P value & $6 \mathrm{M}(\%)$ \\
\hline Surgery & $32.86 \pm 4.69$ & $61.43 \pm 19.56$ & 5.316 & $<0.001$ & $7.00 \pm 0.31$ & $2.92 \pm 0.64$ & 17.717 & $<0.001$ & 85.7 \\
Conservative & $31.76 \pm 3.93$ & $27.64 \pm 17.15$ & 0.965 & 0.347 & $6.82 \pm 0.64$ & $5.79 \pm 0.89$ & 3.775 & $<0.001$ & 35.3 \\
t or $\chi^{2}$ value & 0.706 & 5.123 & & & 0.813 & 9.507 & & 8.016 \\
P value & 0.486 & $<0.001$ & & & 0.422 & $<0.001$ & & 0.005 \\
\hline
\end{tabular}

\section{Discussion and Conclusion}

At present, many scholars agree that: for patients with pathological fractures caused by bone metastasis of malignant tumor, surgical measures are adopted aimed to improve the quality of life of patients during survival rather than prolonging their survival time [3] [4]. But something different is seen to be found in this study. On the one hand, in terms of improving the quality of life, the results of this study confirm the above point of view. In the surgical group, most patients achieved full weight bearing in 2 - 4 weeks after the surgery, resuming a large part of self-care ability. After 2 months of treatment, the KPS score of the survivors was significantly higher than that of the conservative group, and the majority (10/13) was above 60 points, which means that most patients continue to receive standardized oncological radiotherapy, chemotherapy and other specialty treatments. On the contrary, the KPS of the majority of the control group 
is below 40 points, which mean that these patients not only have poor self-care ability, but also difficult to be carried out by standard oncological treatment. On the other hand, we conducted long-term follow-up on all patients after fracture treatment. Survival analysis found that the median survival or 6-month survival rate of patients treated by surgery was higher than that of the conservative group; pathological hip fractures caused by malignant tumor bone metastasis had very poor self-healing ability without surgical intervention [8]. We noticed that only 5 cases of the conservative group achieved partial weight-bearing during the observation period, and most of them died $2-3$ months after the fracture. There may be two reasons contributed to the bad result of conservative treatment: first, long-term bed rest is prone to a variety of complications, such as pneumonia, decubitus, etc., which accelerates the death of patients with malignant tumors and hip fractures; second, patients with conservative treatment of hip fractures are difficult to take care of themselves, and the KPS is so low that the original oncology treatment cannot be performed. This means that good limb function reconstruction is not only the need to improve the quality of life of these patients, but also the necessary means to ensure the successful implementation of oncology treatment, maintain the life expectancy and even prolong to the survival period.

Various surgical reconstruction measures are available to the proximal femoral pathological fracture, including internal plate fixation, intramuscularly nail fixation, and endoparasitic reconstruction, etc. [9] [10] [11] [12]. The prerequisites for successful internal fixation of the steel plate are very stringent. First, there must be enough cortical bone, so that enough stability can be obtained after the operation to allow early weight bearing; second, the joints must be complete and offer sufficient range of motion without pain during everyday activities. These are more difficult to implement for patients with malignant tumors, which greatly limit the application of a steel plate in these cases. Even if the above conditions are met, the place still has an internal fixation failure rate of more than $20 \%$ [13], and the second time operation is more complicated and the patient is poorly tolerated. Now the plate is more inclined to preventive fixation of bone metastatic cancer [14]. intracellular fixation has better photomechanical advantages than steel plates, but two aspects need to consider: firstly, the process of nail placement can easily bring tumor cells to the distal side, causing new metastases and may led to another fracture [12]; secondary, it needs to balance the scraping of the tumor with the preservation of bone to obtain sufficient support, otherwise, little support will easily cause the stress to concentrate on the intermediate nail, leading to the loosening or even breaking of intramuscularly nail. Regardless of the plate or intramuscularly nail fixation, for patients with malignant tumors, the problem of slow healing or even non-healing must be considered [8], which will make it difficult for patients to fully relieve their pain and fail to achieve the purpose of surgery. Artificial enterolithiasis reconstruction is favored by clinicians because of its advantages of faster pain relief. Little needs to focus on bone preservation problems, and early weight-bearing allowance. Many 
scholars regard it as the most suitable functional reconstruction method for pathological fractures of the proximal femur caused by metastasis of malignant tumors [9] [12] [15] [16]. In this study, all the operation of the surgical group was successfully finished. Except for one patient who died of multiple organ failure in a short period of time, all other patients went back to the ground within 1 week and achieved full weight bearing within 2 - 4 weeks. MSTS average score is $20.38 \pm 0.93$ for the 13 survivors, reaching about $70 \%$ of normal limb function and meeting the need for self-care. Furthermore, the operation is safe and can be tolerable for most patients.

Although endocarditis reconstruction has advantages for these patients, the potential risks should also be obvious. The serious complications, which are disastrous, are loosening of the prosthesis and infection in the long-term [12]. These complications were not found in this study for two possible reasons: firstly, the sample size of this study is too small, and secondary, the survival time of the cases in this study was generally short, did not reaching the time of the above complications. It needs to be noticed that prosthesis dislocation and gait abnormalities are more likely to occur after prosthesis replacement due to extensive tissue dissection, injury, and resection [17]. No dislocation cases have been seen in this study, but 1 case of postoperative gait abnormalities is observed, which is worth noting. We believe that this type of reconstruction surgery has the following main points: 1) Pay attention to the improvement of preoperative examination, preoperative CT or MR can better help the planning of the tumor segment resection range, but it is still difficult to determine the resection range and the size of the prosthesis very accurately, so It is necessary to prepare multiple models of prostheses. And the preoperative design of $3 \mathrm{D}$ printing assisted personalized prosthesis is more advantageous in this regard and worth further study. 2) The resection of the tumor segment should be able to include a small amount of normal bone tissue to reduce the local recurrence rate [18], and normal bone tissue is conducive to early bonding of the prosthesis and bone and are beneficial to avoid the occurrence of fractures around the prosthesis. 3) Using the bone cement properly. Bone cement can be used as an adhesive to fix the prosthesis in a short period of time during tumor prosthesis replacement, and can also be used as a filler to make up for the defective bone, especially when the pelvis and acetabulum were invaded so that it is need to be scraped. Bone cement can greatly increase the stability of the prosthesis. In addition, the exothermic effect of bone cement solidification also has a certain killing effect on residual tumor cells, reducing local recurrence [19]. In this study, bone cement was used as a filler to fill the cavity after acetabular lesions were scraped in 6 cases. After surgery, the patients can still go to the ground normally and birth weight, and the effect is satisfactory. 4) Pay attention to the protection and reconstruction of soft tissue. When it is inevitable to remove part of the invaded soft tissue, try to leave the suture joint capsule as far as possible. If it cannot be left, postoperative orthopedic shoes or braces should be fixed. The method adopted by Guo Wei et al. [20] show the gluteus medius and gluteus minor ten- 
dons to the great trochanter of the prosthesis are worth learning. It is necessary to overlap and tightly stitch the remaining abductor muscles with the lateral femoral muscles to form a "sleeve" to protect the proximal prosthesis and maintain tension.

\section{Conflicts of Interest}

The authors declare no conflicts of interest regarding the publication of this paper.

\section{References}

[1] He, Z.S., Bai, J.P., et al. (2010) Surgical Treatment of Pathological Fracture in $20 \mathrm{~Pa}-$ tients with Proximal Femoral Metastatic Tumor. Chinese Journal of Bone Tumor and Bone Disease, 9, 492-494.

[2] Jacofsky, D.J. and Haidukewych, G.J. (2004) Management of Pathologic Fractures of the Proximal Femur: State of the Art. Journal of Orthopaedic Trauma, 18, 459-469. https://doi.org/10.1097/00005131-200408000-00013

[3] Calabro, T., ROOyen, R.V., Piraino, I., et al. (2016) Reconstruction of the Proximal Femur with a Modular Resection Prosthesis. European Journal of Orthopaedic Surgery and Traumatology, 26, 415-421. https://doi.org/10.1007/s00590-016-1764-0

[4] Wang, F., Yang, X.G., Liu, Y.H., et al. (2017) Effectiveness of Modular Intercalary Endoprosthesis for Femoral Metastatic Pathological Fracture. Chinese Journal of Geriatric Orthopaedics and Rehabilitation (Electronic Edition), 3, 197-204.

[5] Peterson, J.R., Decilveo, A.P., O'connor, I.T., et al. (2017) What Are the Functional Results and Complications with Long Term Hemiarthroplasty in Patients with Metastases to the Proximal Femur? Clinical Orthopaedics and Related Research, 475, 745-756. https://doi.org/10.1007/s11999-016-4810-7

[6] Zoccali, C., Attala, D., Uccio, A.S.D., et al. (2017) The Dual Mobility Cup in Muscular Skeletal Oncology: Rationale and Indications. International Orthopaedics, 41, 447-453. https://doi.org/10.1007/s00264-017-3407-4

[7] Van Der Linden, Y.M., Dijkstra, S.P., Vonk, E.J., et al. (2005) Prediction of Survival in Patients with Metastases in the Spinal Column-Results Based on a Randomized Trial of Radiotherapy. Cancer, 103, 320-328. https://doi.org/10.1002/cncr.20756

[8] Sarahrudi, K., Grdtbauer, M., Piatzer, P., et al. (2009) Surgical Treatment of Metastases Fractures of the Femur: A Retrospective Analysis of 142 Patients. The Journal of Trauma, 66, 1158-1163. https://doi.org/10.1097/TA.0b013e3181622bca

[9] Harvey, N., Ahlmann, E.R., Auison, D.C., et al. (2012) Endoprostheses Last Longer than Intramedullary Devices in Proximal Femur Metastases. Clinical Orthopaedics and Related Research, 470, 684-691. https://doi.org/10.1007/s11999-011-2038-0

[10] Wang, S.F. and Xiong, J. (2009) Progress in the Treatment of Metastatic Carcinoma of the Proximal Femur. Chinese Journal of Bone Tumor and Bone Disease, 8, 370-375.

[11] Wang, S.F., Xiong, J., Luo, D.S., et al. (2009) Surgical Management of Metastasis in Proximai Femur. Journal of Practical Orthopaedics, 15, 895-898.

[12] Zhang, Y.S. and Shang, G.N. (2018) Surgical Treatment of Pathological Fracture of Long Bone Metastases of Lower Extremities. Chinese Journal of Surgical Oncology, 10, 77-80.

[13] Yazawa, J., Frassica, F.J., Chao, E.F., et al. (1990) Metastatic Bone Disease. A Study 
of the Surgical Treatment of 166 Pathologic Humeral and Femoral Fractures. Journal of Clinical Orthopaedics, 251, 213-219.

https://doi.org/10.1097/00003086-199002000-00036

[14] Yang, Y., Yang, Y.L. and Guo, W. (2011) 59 Cases of Retrospective Analysis of Surgical Treatment of Humeral Metastatic Carcinoma. Journal of Peking University (Medical Edition), 43, 681-685.

[15] Steensma, M., BoIand, P.J., Morris, C.D., et al. (2012) Endoprosthetic Treatment Is More Durable for Pathologic Proximal Femur Fractures. Clinical Orthopaedics and Related Research, 470, 920-926. https://doi.org/10.1007/s11999-011-2047-Z

[16] Guzik, G. (2016) Treatment Outcomes and Quality of Life after the Implantation of Modular Prostheses of the Proximal Femur in Patients with Cancer Metastases. Ortopedia Traumatologia Rehabilitacja, 18, 231-238. https://doi.org/10.5604/15093492.1212867

[17] Chen, K., Liang, W.Q. and Cai, Z.D. (2010) Endoprosthetic Proximal Femoral Replacement for 37 Patients with Proximal Femoral Metastatic Tumor in One Institute: Retrospective Analysis within One Year. Journal of Clinical Rehabilitative Tissue Engineering Research, 14, 5551-5554.

[18] Zhang, W.B. (2008) Treatment Strategies for Bone Metastatic Tumors. Journal of Practical Oncology, 23, 290-293.

[19] Houdek, M.T., Watts, C.D., Wyles, C.C., et al. (2016) Functional and Oncologic Outcome of Cemented Endoprosthesis for Malignant Proximal Femorai Tumor. Journal of Surgical Oncology, 114, 501-506. https://doi.org/10.1002/jso.24339

[20] Guo, W., Yang, Y., Tang, X.D., et al. (2007) Endoprosthetic Reconstruction after Resection of the Tumor of the Proximal Femur. Chinese Journal of Surgery, 45, 657-660. 